“C 2017 IEEE. Personal use of this material is permitted. Permission from IEEE must be obtained for all other uses, in any current or future media, including reprinting/republishing this material for advertising or promotional purposes, creating new collective works, for resale or redistribution to servers or lists, or reuse of any copyrighted component of this work in other works." 


\title{
Would You Like to Sample? Robot Engagement in a Shopping Centre
}

\author{
Meg Tonkin ${ }^{1}$, Jonathan Vitale ${ }^{1}$, Suman Ojha ${ }^{1}$, Mary-Anne Williams ${ }^{1}$, \\ Paul Fuller ${ }^{2}$, William Judge ${ }^{3}$ and Xun Wang ${ }^{3}$
}

\begin{abstract}
Nowadays, robots are gradually appearing in public spaces such as libraries, train stations, airports and shopping centres. Only a limited percentage of research literature explores robot applications in public spaces. Studying robot applications in the wild is particularly important for designing commercially viable applications able to meet a specific goal. Therefore, in this paper we conduct an experiment to test a robot application in a shopping centre, aiming to provide results relevant for today's technological capability and market. We compared the performance of a robot and a human in promoting food samples in a shopping centre, a well known commercial application, and then analysed the effects of the type of engagement used to achieve this goal. Our results show that the robot is able to engage customers similarly to a human as expected. However unexpectedly, while an actively engaging human was able to perform better than a passively engaging human, we found the opposite effect for the robot. In this paper we investigate this phenomenon, with possible explanation ready to be explored and tested in subsequent research.
\end{abstract}

\section{INTRODUCTION}

The robots are coming. Only in some places, however, are they genuinely already here [1], [2]. These early days of adoption into society of social robots are an opportunity but also an indication for social roboticists and human robot interaction researchers that it may be time to move experiments as much as possible out from the lab into the wild[3], despite the difficulties that this entails.

While the future may hold robots that seamlessly interact in a social manner with humans and can perform any multitude of social and cognitive tasks, in the present we are still limited in the level of performance that is achievable and practical [4]. As social robots are being placed in public spaces today, we need research and research methodologies that are relevant for today's environment and valid roles for robots to perform.

Previous work concerning robots in shopping centres has established the effectiveness of robots for attracting attention of people and potential roles such as shopping assistants, information guides and event promotion [5], [6], [7]. However, when a robot is situated in a public space and required to achieve a specific goal, attracting human attention

\footnotetext{
${ }^{1}$ Meg Tonkin, Jonathan Vitale, Suman Ojha and Mary-Anne Williams are with Faculty of Engineering and Information Technology at the Centre For Artificial Intelligence - Innovation and Enterprise Research Lab (The Magic Lab), University of Technology Sydney, 15 Broadway, Ultimo NSW 2007, Australia. Primary contact: margaret.tonkinestudent.uts.edu.au

${ }^{2}$ Paul Fuller is with Stockland, 133 Castlereagh Street Sydney 2000, Australia. E-mail: paul.fullerestockland.com.au

${ }^{3}$ William Judge and Xun Wang are with Commonwealth Bank Innovation Lab, 11 Harbour St, Sydney NSW 2000, Australia. E-mail: william.judge@cba.com.au
}

is not sufficient. Social interactions in public environments are particularly complex and unpredictable [8]. Thus, other factors can impact on the final outcome. These factors may not be discovered in laboratory settings [9]. Hence, it is of paramount importance to investigate these phenomena by designing experiments that simulate as much as possible the natural conditions occurring everyday in the selected public space. Therefore, we aim to fill this research gap by investigating roles a robot can perform attracting attention to a desirable effect, providing results that are of significance for all stakeholders, and importantly business, customers and researchers.

Robots in public spaces, especially those with plausible roles such as receptionists, shopping assistants and tour guides, all present with the same challenge - that of interacting with humans using social behaviour to perform their task [3], [10]. Since the study of human robot interactions (HRI) is a complex and vast field, in this paper we narrow our focus to a specific component within an interaction, that of social engagement. Engagement may be considered an essential element of a successful experience of human robot interaction, a "characterizing feature of the quality of the experiences with social robots" [11].

Engagement in HRI is commonly measured via social behaviours displayed by humans during an interaction, such as head movements, gaze, joint attention and body posture [12] as indicators of the level of engagement and also with surveys for users to confirm their engagement [13]. This paper is novel in our approach by applying an application allowing the social engagement to be measured via the performance of the robot in a naturalistic interaction task and setting. For example, instead of the robot performing an interaction and asking users to complete an interview afterwards as to their engagement, we instead operationalise our measurement. We define our user engagement by the users investment in a physical action in the engagement process, similarly to evaluating user engagement on a website via number of physical user actions such as click throughs [14]. Therefore, we chose a common task prevalent in shopping centres: that of inviting shoppers to try a sample of food. This task is regularly perceived and understood by shoppers, and it has an engagement model that involves a physical user action in the process - that of taking a sample. This method of engagement measurement minimises the impact of the experimental design on natural conditions encountered everyday in a public place such as a shopping centre, thus allowing the discovery of additional unforeseeable phenomena and may provide many more insights for designing and 
developing successful commercial robot applications.

Our approach for the task engagement model incorporated verbal and non verbal engagement behaviours such as speech, gesturing and head movements. The task measured not the level of engagement by shoppers but whether the model for the engagement process successfully achieved the goal of giving away samples. Additionally, we set the task benchmark by comparing a human in the same role to the robot. As such, the main objective of this paper is to compare the performance of a robot and a human in promoting food samples in a shopping centre, using different engagement models. Essentially asking the question to what extent can a robot perform a task requiring social engagement as well as a human?

In this paper we will show that a robot can perform the task at hand in a comparable way to a human but it presents some limitations in engaging humans when in an actively interacting role. We conclude that collaboration between a robot and human, with the robot operator in the job of attracting shoppers and initiating engagement and the human operator working to maintain engagement, may be a sensible way forward for future research and a viable commercial robot application.

The sections of this paper are organised as follows: Section II describes related works in naturalistic settings and studies on robots' social engagement, Section III presents engagement and its role in social robotics, Section IV provides the methodology used in our study, Section $\mathrm{V}$ gives the results and analysis of the experimental data and Section VI discuss our conclusions and the aim of future work.

\section{RELATED WORKS}

Although being advocated since the advent of robots, reported scientific studies in the wild are relatively few, particularly in HRI [3], [15]. However a step change may be observed to be occurring [16], [17].

Conducting human-robot interaction experiments in naturalistic environments like shopping centres, museums and airports possesses issues usually not encountered in experiments in the lab [6], [18], [19]. Robots that perform well in lab environments are typically not as successful in naturalistic environments which introduce more variables and a larger variety of social phenomena [3]. For example, it has been shown that the environment in which a robot is situated has a significant effect on the human-robot social interaction [20]. Furthermore, social interaction and engagement is subtle, people make snap judgements - decisions in microseconds that determine whether or not they like or dislike someone [21]. Moreover, what is in scarce supply in research is methodology for deployment and publications highlighting things that go wrong [15]. Hence, it is of paramount importance to test the robot application "in the wild" [22], [3]. By saying "wild" we mean environments introducing significant challenges for the robot, namely public areas where there is a likely presence of complex social interactions.

Our study focuses on engagement, a topic currently not sufficiently covered by HRI experiments in public environ- ments. Datta, Kapuria and Vijay [10] showed that the type of interactions executed by the robot in a shopping mall impacts on the level of engagement obtained from the people. Other human-robot interaction experiments in public spaces suggested that robots making use of social cues like speech, gaze or gestures can better engage people in interaction [23]. Similarly, the study by Sidner et al. [24] found that a penguin robot that performed only a few gestures was proved to be more engaging than the one which did not move at all. Contrasting movement vs no movement, while rudimentary, highlights movement is key, and a direction for further exploration, including in this paper. Moreover, Nass and Gong [25] proposed that as the degree of social cues of a robot in a public space increased, the engagement of the people interacting also increased. These findings suggest that a robot actively interacting with people is more capable of maintaining the engagement of humans in naturalistic environment.

Prior works studying engagement in human-robot interactions [25], [24] investigate engagement by measuring social interaction cues (e.g. gazing, attention, etc. ) or by employing questionnaires. While of utmost importance there is no direct analysis of engagement impacting on the real objective desired for the robotic platform. Filling this gap is particularly beneficial to foster commercial applications of robotic platforms. Therefore, in this work, we begin to address this gap.

\section{ENGAGEMENT AND ITS ROLE IN SOCIAL ROBOTICS}

Engagement is commonly defined in human robot interaction studies [12], [13], [11] and for the purposes of this paper, as "the process by which individuals involved in an interaction start, maintain and end their perceived connection to one another" [13].

This perceived connectedness may be indicated by verbal and non-verbal behaviours such as joint attention, head and eye movements, gestures and conversational turn taking, and physiological changes that take place during an interaction [11], [26]. As such, the detection and measurement of these behaviours for evaluating engagement in social robotics has been a topic of great interest, and explored for more than a decade [11], [12], [27], [13], [28]. For instance, studies have investigated automatic affect recognition [4], the effect of face tracking [13], directed gaze and mutual facial gaze [12], and sometimes all at once, as in the work by Anzalone et al. [11] where an RGB-D sensor was used to monitor body posture variation, head movements, synchronous events, imitation cues and joint attention, and integrated all the behaviours for analysis. These measurements may also be combined with a follow up user survey on self reported engagement levels [13].

Nevertheless, defining and measuring engagement by only these type of physiological and behavioural metrics does not have to be the only approach, especially in the case of commercial applications. To design the methodology used in this study, we draw inspiration by works in Human 
Computer Interaction (HCI). In on-line services, engagement characteristics are identified as dependant on the application and experience at hand and a viable methodology to assess user's engagement is to measure the number of physical user actions such as click throughs [14]. Therefore, we propose a similar methodology for human robot interaction. Factors which affect engagement are multitude and diverse. There are individual user factors, such as personality traits and social attitudes [26], situational and task factors [29], [4], and environmental factors, such as the distance between individuals [27], to name but a few. Hence, of supreme importance when in the wild, is to measure engagement with a specific purpose or application in mind. As such, in this paper, we focus on measuring user engagement by the successful completion of the application goal, rather than affects displayed by shoppers. While a simplistic measure, for assessing commercial application relevant for business it enables vital comparison of performance.

For a better analysis of the topic, we compared two engagement models: passive engagement and active engagement, as based on fulfilling needs in a public space [30]. In our passive engagement model the operator does not move from the one spot, does not actively interact with shoppers and is behind a rope barricade, allowing shoppers to simply observe the operator. In this setting the robot is programmed to perform autonomous random interactions and the human acts disengaged, almost robotically, as described by Shi and colleagues [16]. In the active model, the operator can actively engage with shoppers in a timely fashion and move around. In this second setting the robot operator is teleoperated to simulate an intelligent social agent able to directly interact with shoppers in a timely manner, comparable to the human operator. Our model is illustrated in Fig. 1, where the operator is represented as the square body, and shoppers as round bodies. The operator is trying to achieve the goal of distributing samples. This is a social task requiring to first engage any shoppers passing by. Therefore, the operator gestures out into public space for initiating engagement. However, just attracting shoppers' attention is not sufficient to fulfil the goal of the task. It is necessary to motivate shoppers to action, i.e. motivating them to take a chocolate sample. Thus, the operator directs shoppers' attention to the table containing the samples. Finally, the engagement is terminated by gesturing and facing away from the shopper. A protocol was created for both the robot operator and the human operator to follow and this, along with our hypotheses, is described in the following section.

\section{METHODOLOGY}

Given our objective, to compare the performance of a robot and a human in promoting food samples and to analyse the effects of the model of engagement used to achieve this goal, we propose the following hypotheses:

Hypothesis 1 The robot operator is able to engage and motivate people to take a sample with comparable or better performance than a human operator.

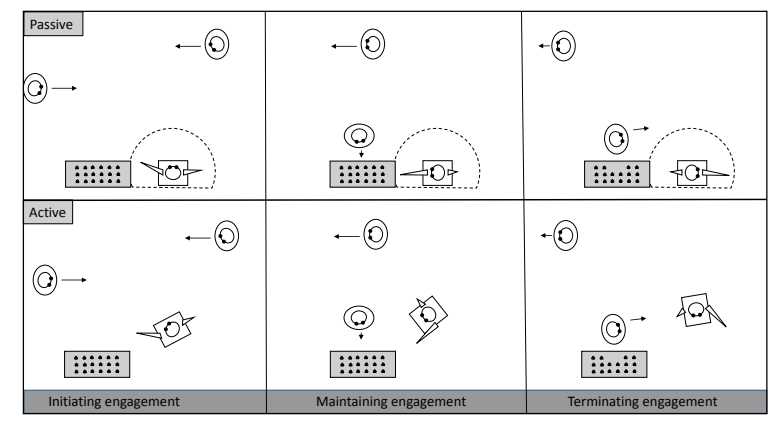

Fig. 1. The engagement model as discussed in this work. On the top row: passive engagement setting without any direct interaction by the operator and remaining in one spot. On the bottom row: during the active engagement setting the operator is able to directly interact and move slightly towards shoppers. In both models engaged shoppers move to the table and take a sample, non-engaged shoppers continue past.

Hypothesis 2 An active engagement model significantly increases number of samples taken compared to a passive engagement model.

To test our hypotheses, we designed an experiment considering two binary variables: the operator promoting the chocolate samples (i.e. robot or human) and the type of engagement used (i.e. passive engagement or active engagement). This design led to four experimental settings: a passively engaging robot operator, an actively engaging robot operator, a passively engaging human operator, and an actively engaging human operator.

We will assess the proposed hypotheses by analysing the experimental data as described in the remainder of this section.

\section{A. Operators}

During the robot operator settings we used a PAL REEM humanoid service $\operatorname{robot}^{1}$. The robot is wheel-based and equipped with a pair of stereo cameras on its head as eyes, with laser and ultrasound scanners for navigation. The robot also has a pair of 4-degrees of freedom (DOF) arms and 7-DOF hands that can perform human-like gestures (Fig. 2).

The human operator used during the other settings of this study was a female English native-speaker, in her thirties, with more than four years sales experience including front of store promotions.

\section{B. Engagement Protocols}

We designed protocols for the active and passive engagement settings. During the passive engagement setting, the protocol required the operator (both robot and human) to adhere to a specific set of gesture and speeches. The speech employed was randomly selected from a pre-defined list of possible options. The actions of the passive operator were executed in a specific order as per the following three steps:

1) Initiation of Engagement. In this step the operator attracts the attention of customers. The robot head turned

\footnotetext{
${ }^{1}$ http://pal-robotics.com/wp-content/uploads/2016/03/REEMDatasheet.pdf
} 


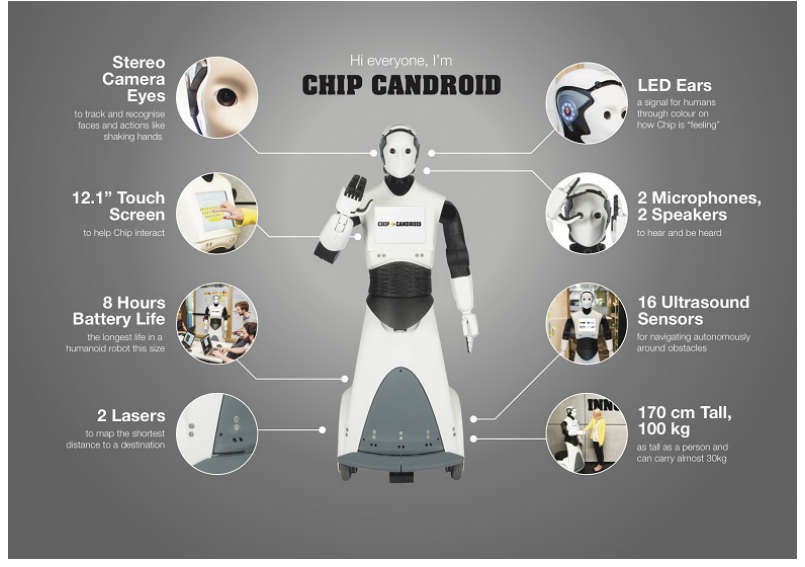

Fig. 2. The robotic platform used in our study.

from side to side, appearing to look out over the study area, scanning the environment. One arm was raised and waved. The randomly selected speech included welcoming sentences such as "Welcome shoppers! This is your lucky day!";

2) Maintaining Engagement. In this step the operator promotes the chocolates. The arm was lowered and pointed towards chocolates, the robot head was turned downwards gazing towards the chocolates, to bring user attention to the chocolates. The speech included invitations to taking a free chocolate (e.g. "Life is better with a chocolate, please have one!');

3) Termination of Engagement. In this step the operator ends the interaction. The robot head and torso were turned to facilitate the arm to gesture towards a nearby survey/exit area. The speech included thanks and concluding sentences (e.g. "Thank you! Please, take a survey as you leave").

Between each step the robot was programmed to wait approximately 10 seconds. Importantly, during the passive engagement setting the operator did not move from its/her location and its/her behaviour was not synchronised with customers approaching or leaving the study area. We asked the human operator to refrain from specifically making eye contact with the shoppers crossing the experiment area and asked her to not directly interact with shoppers, for example if a shopper asked for directions. When this scenario occurred, a researcher was instructed to appear and interact with the customer instead, while the human operator continued to act passively as per the designed protocol.

On the contrary, during the active engagement setting, the operator was free to directly interact with the approaching shoppers, although still adhering to the three steps previously described (i.e. initiation, maintenance and termination). Specifically, the robot was teleoperated to simulate an intelligent social agent able to interact with shoppers. Its base was rotated and moved so to direct its face to the approaching shoppers and to briefly move towards them. The robot was programmed to attract shoppers by using the welcoming sentences and by communicating to the shoppers to interact by gesturing with its arm. Therefore, given the experiment was taking place in the wild and the ability of the robot to interact with people, one of the most common occurrences was for people to ask for a selfie with the robot. In that case, the robot answered positively and posed for a selfie with the shoppers.

\section{Location and Time of the Study}

The study was conducted in a two level shopping centre containing over 150 retail stores, with a strong community of regular shoppers. The robot was introduced in this shopping centre a few days before our study during a public event. The event was recorded and presented on television during a major Australian news broadcasting. Thus, shoppers should have had the opportunity to see the robot, although they were not exposed to the task considered in this study, namely providing samples to shoppers. The setting consisted of a bounded area. During the passive engagement setting we used a rope barricade around the operator. This barricade was removed in the active engagement setting.

Due to technical constraints and agreements with our commercial partner (i.e. space available for the study, availability of the robot at the shopping centre and supported length of the study), the study was conducted on the second floor of the shopping centre during two separate week days. For each day, we set up two experimental settings in two separate time slots of 1.5 hours each:

1) Day one. Robot operator settings: passively engaging setting from 9.45am to $11.15 \mathrm{am}$, actively engaging setting from $12.45 \mathrm{pm}$ to $2.15 \mathrm{pm}$;

2) Day two. Human operator settings: passively engaging setting from $9.45 \mathrm{am}$ to $11.15 \mathrm{am}$, actively engaging setting from $12.45 \mathrm{pm}$ to $2.15 \mathrm{pm}$;

The selected days and time slots did not affect the number of potential shoppers available for our study. In fact, previous and post-study statistics showed a comparable number of customers during the selected times and days. However, our choice had an effect on the type of shoppers visiting the shopping centre. In fact, during morning/early afternoon of week days the most frequent customers of the shopping centre are mums with small children and elderly people. However, this was not a limitation for our study but rather a benefit; in fact, the selected times resemble the ones that are usually available for promoting food in the shopping mall, thus providing a good opportunity to test the effects of a robotic platform under circumstances similar to real commercial situations.

\section{Measurements}

The main objective of this paper is to compare the performance of a robot and a human operator in promoting food samples in a shopping centre and to analyse the effects of the type of engagement used to achieve this goal.

Free sample promotions can increase store sales on the day of the promotion [31], hence a promoter able to give away as many samples as possible, to different customers, is of benefit. Therefore, we counted the number of distinct 
TABLE I

NUMBER OF GIVEAWAY SAMPLES DURING THE INVESTIGATED SETTINGS

\begin{tabular}{l||ccc} 
& Human & Robot & Outcome \\
\hline \hline Passive & 30 samples & 46 samples & $+53 \%$ \\
Active & 50 samples & 31 samples & $-38 \%$ \\
Outcome & $+66 \%$ & $-32 \%$ &
\end{tabular}

customers the operator was able to engage via the number of samples taken. Accordingly, if the shopper took more than one chocolate (for example to distribute them to other customers that did not approach the operator) we counted only the first pick-up and we did not count people approaching the operator but not taking any sample (as illustrated in Fig. 1).

To complement the considered measure, we recorded the time in which each sample was taken by the customers, and therefore the delays between each measurement. This helped us to estimate the average speed by which the operator was able to fulfil the assigned task.

The measurements were taken by an instructed researcher able to observe the experimental area and note the samples distributed in a smartphone application. The customers were not aware of the samples taken being counted.

\section{RESULTS}

Table I provides the number of samples given away to distinct shoppers during the considered settings. In agreement with our predictions, during the passive engagement condition, the robot performed better than the human by increasing the number of giveaways by $53 \%$. However, contrary to what was predicted, the robot was not able to perform better than the human operator during the active engagement condition. In this setting the robot decreased the number of giveaways by $38 \%$. Surprisingly, a robot operator actively engaging people resulted in a negative trend compared to a passive robot, with $32 \%$ less giveaways. On the contrary, the actively engaging human performed better compared to the passive engagement setting by resulting in a positive trend of $66 \%$, as we expected.

Beside comparing the overall number of people taking a sample, we analysed the speed of execution of the considered task throughout the duration of each setting. Therefore, we divided the total duration of each setting (5400 seconds) into time slots of 200 seconds, thus obtaining 27 'checkpoints'. For each checkpoint, we computed the number of measurements (i.e. the number of distinct users that took a sample) up to that time. This process led to 27 new measurements for each of the 4 settings. By denoting the sampled measurements with $Y_{\text {sampled }}$ and the time checkpoints with $X$, it is possible to explain the data via the following linear model:

$$
Y_{\text {sampled }} \approx b+m X
$$

where $b$ is the $y$-axis intercept of the line (initial velocity) and $m$ is the gradient of the line (speed). In this analysis we are interested in the average speed to execute the required task. Therefore, to more easily compare the speed between the

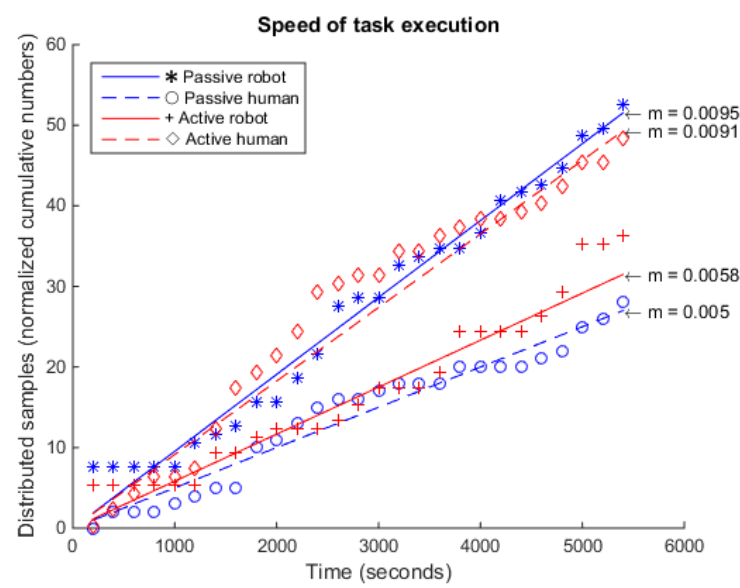

Fig. 3. Result of the linear regression process over the sampled measurements. The gradients of the lines represent the average speed of executing the task. To a higher gradient it corresponds a higher speed.

considered 4 settings we subtracted the initial velocity factor $b$ from the gathered samples, thus obtaining the following normalised linear model:

$$
\bar{Y}_{\text {sampled }} \approx m X
$$

where $\bar{Y}_{\text {sampled }}=Y_{\text {sampled }}-b$. We estimated the parameters $b$ and $m$ for each setting by using the Theil-Sen estimator [32], which is a linear regression algorithm robust to outliers. Fig. 3 illustrates the results of the linear regression plotted as per the linear model in (2). The passive robot shows the higher average speed, followed by the active human with comparable performance. On the contrary, the active robot and the passive human show dramatically worse performance, with the passive human resulting marginally slower than the active robot.

A two-way ANOVA was run on the gathered samples $\bar{Y}_{\text {sampled }}$ with a confidence level of $95 \%$ to examine the effect of type of engagement and operator on the number of samples gave away over time. There was a significant interaction between the effects of type of engagement and operator, $F(1,104)=22.46, p=.0000$. No main effects were observed between passive and active engagement $(\mathrm{p}=.5148)$ and robot or human operator $(\mathrm{p}=.5371)$. We measured the effect size of the observed interaction via partial eta squared [33] suggesting a large effect size $\left(\eta_{p}^{2}=.1776\right)$ [34].

In order to further analyse the observed interaction effect we ran a one-way ANOVA on the gathered samples $\bar{Y}_{\text {sampled }}$ to examine the effects between the 4 considered settings. This process led to identify a significant effect between the 4 settings, $F(3,104)=7.76, p=.0001$ with large effect size $\left(\eta^{2}=.1828\right)$ [34]. A Tukey-Kramer multiple comparison was run as post-hoc analysis to assess the significant effects between the 4 settings. Similarly to what suggested by Fig. 3, the passive robot resulted significantly faster than the passive human $(\mathrm{p}=.0014)$ and than the active robot $(\mathrm{p}=.0240)$, whereas the active human resulted significantly faster than the passive human $(\mathrm{p}=.0013)$ and than the active robot $(\mathrm{p}=$ .0224). No effects were detected between the passive robot 
TABLE II

DESCRIPTIVE STATISTICS OF THE COLLECTED TIME DELAYS.

\begin{tabular}{lccc}
\hline Setting & Mean & Median & Std \\
\hline Passive robot & $119 \mathrm{sec}$ & $78 \mathrm{sec}$ & $166 \mathrm{sec}$ \\
Passive human & $180 \mathrm{sec}$ & $132 \mathrm{sec}$ & $207 \mathrm{sec}$ \\
Active robot & $178 \mathrm{sec}$ & $31 \mathrm{sec}$ & $293 \mathrm{sec}$ \\
Active human & $106 \mathrm{sec}$ & $70 \mathrm{sec}$ & $127 \mathrm{sec}$ \\
\hline
\end{tabular}

and the active human $(\mathrm{p}=1)$ and between the passive human and the active robot $(\mathrm{p}=.8048)$.

In summary, we observed that:

1) The passively engaging robot performed in a comparable way or better than the human operator for both passive and active engagement conditions. This suggests that a passive robot can engage customers and promote food samples in a comparable way to a human operator, thus validating our first hypothesis;

2) As expected, the actively engaging human performed significantly better than the passive human. However, surprisingly the actively engaging robot performed significantly worse than the passive robot and with comparable performance with respect to the passive human. These results suggest that the active engagement was beneficial only for the human operator and apparently detrimental for the robot operator, thus only partially validating our second hypothesis.

In the following section we will provide further analyses to propose a potential explanation for the second unexpected result.

\section{A. Group Influence}

The speed to execute the desired task is directly related to the time delays between each giveaway. In other words, the longer the delays between each giveaway, the slower is the observed average speed.

Therefore, to deepen the analyses proposed in the previous section, we started by analysing the descriptive statistics of the measured time delays between giveaways during the 4 considered settings. The descriptive statistics are summarised in Table II.

The computed means are consistent with the previously discussed results. In fact, the passive robot and active human have comparable average time delays, which are significantly lower than the passive human and active robot conditions showing comparable worse performance. However, although the active robot and passive human presents comparable means, their medians are profoundly different. More surprisingly, the computed median for the active robot results in the lowest statistic compared to the other settings and it is less than a half of the second best median (70 seconds during the active human condition). In addition, the standard deviation for the active robot condition is dramatically higher than the other settings.

This first analysis suggests that, whereas the passive human did in general a relatively poor job in distributing samples to the shoppers since both mean and median resulted worse than the ones in the other settings, the active robot
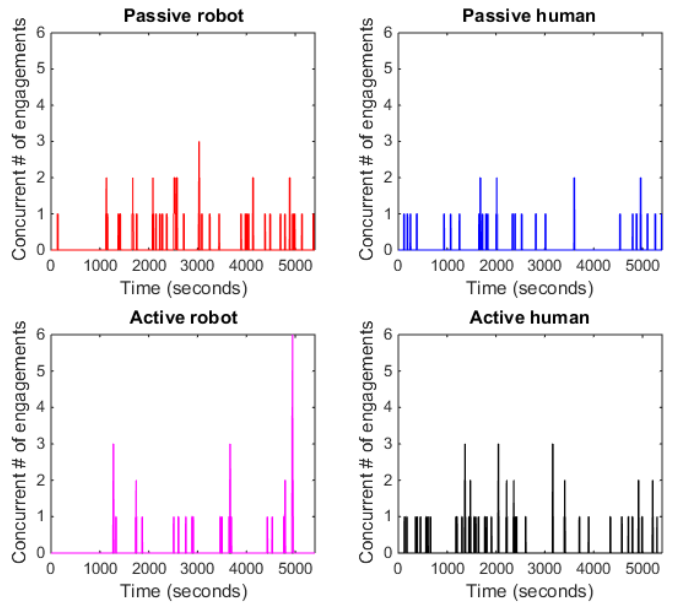

Fig. 4. Estimated number of shoppers taking a chocolate at similar times ( \pm 7 seconds).

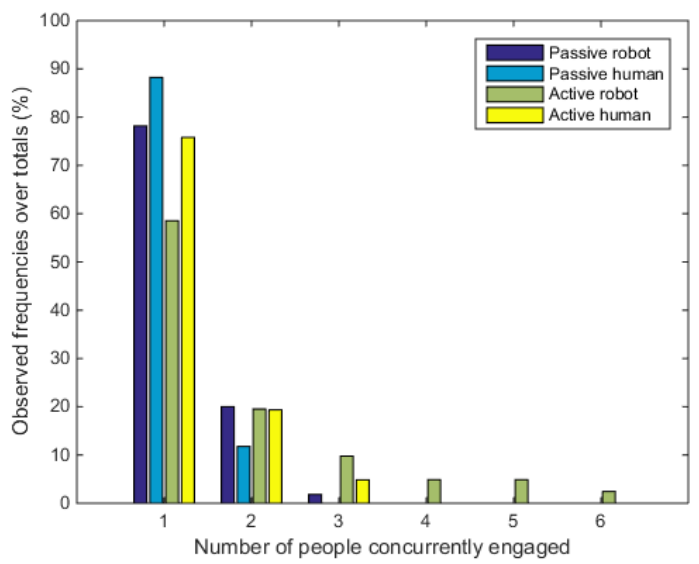

Fig. 5. Frequencies of shoppers taking chocolates at close times $( \pm 7$ seconds) during each setting.

was governed by periods of short delays able to reduce the median alternated by long periods of time able to bias the overall mean. Why did this happen?

Based on our observations in the field, our suggestion is that the active robot was able to attract groups of people rather than single shoppers and to maintain the customers' engagement for a longer period of time. When this happened, it created a situation in which many shoppers took the chocolates with short time delays. After the group of people left the experimental area, the active robot took a while before being able to engage another group of people.

To confirm this analysis we estimated from our measurements the number of shoppers taking a sample at the same time. Given a time window of \pm 7 seconds, we estimated the number of shoppers taking a chocolate at approximately the same time by counting how many measurements occurred during overlapping time windows. The results are illustrated in Fig. 4 and Fig. 5.

From Fig. 4 it is clear to see that the active robot, contrary to the other conditions, is governed by longer periods of time 
with no customers taking samples. Furthermore, the active robot presents higher peaks of shoppers taking samples at approximately same time compared to the other settings. From Fig. 5 it is possible to compare the frequencies of estimated numbers of shoppers taking samples at the same time. Clearly, the active robot is able to attract and distribute samples to larger groups of people compared to the other conditions. However, the active human too was able to attract more frequently larger group of people compared to the passive conditions.

We propose that this phenomenon can be explained by the risk potentially perceived by shoppers in engaging with the operator and taking the sample. In our scenario, when customers pass through the experimental area they can take a decision: engage with the operator and therefore take a sample or ignore the promoter and leave the area. Taking the decision of engaging with the operator underlies many potential risks, such as wasting time, getting embarrassed due to other shoppers observing the situation, feeling obliged to buy the product after trying it, etc. Whenever a decision is taken under uncertain circumstances, like in this study, people need to assess the situation and decide whether to take the related risk or not [35]. Psychology studies suggests that the perception of risk can be reduced when taking a decision in a group compared to taking the same decision alone [36]. This effect is known by the name "group shift" or "risky shift" [37].

Since during the active engagement conditions the interactions last longer compared to the passive engagement conditions, there are more chances for customers to observe a group of people engaging with the operator. Therefore, given the previous discussion, observing a group of people engaging with the operator in the experimental area can likely influence the perception of risk of other shoppers passing through the study area and consequently increase the chance of engaging them.

This analysis can explain why during the active engagement conditions we observe more people taking samples at the same time, which in itself may be considered a form of social engagement, but it does not explain why the active robot shows longer periods of times between each engagement. To explain this second phenomenon recall that the considered measurements included only the customers that approached the operator $A N D$ took a sample. During the active robot condition we observed that many shoppers approached the robot to interact with it and take selfies but they did not take the chocolate sample, thus not being counted in our measurements. In other words, the active robot was able to attract people similarly to the active human (step 1, initiation), but it was not able to correctly manage dyadic situations for distributing the chocolate samples (step 2 , engagement maintenance) probably because the simulated intelligent capabilities of the robot distracted people from the real purpose of the robotic application, which it was not to interact with users, but to promote food samples.

An actively engaging and socially intelligent robot able to attract larger groups of people led to surprising detrimental performance when distributing samples. This is an important insight, since in this specific activity (and in many other commercial applications requiring customers engagement) it is not important to just attract people, as normally suggested in previous works investigating engagement, but rather to attract people in order to achieve the desired goal, which in this study was to distribute as many samples as possible to distinct individuals.

\section{CONCLUSIONS}

We presented a novel human-robot interaction experiment in a shopping centre to explore the effects of engagement on promoting a commercial robot application.

We discovered that a robot is comparable to a human with the specific task of engaging shoppers and giving away samples. The passively interacting robot consistently attracts individual shoppers and engages with them. In contrast, an actively interacting robot gave away fewer samples in total but may draw a bigger crowd when it manages to do so. Further research is required to identify the factors behind this and possible solutions for future commercial applications.

There are some caveats to this finding. Our engagement model is specific to our task, hence our definition of engagement may not apply to different applications not requiring a physical user action. Additionally, due to our engagement model following a set pattern (initiate, maintain, terminate), we did not take advantage of shoppers asking for a selfie with the robot, i.e. informing shoppers they must take a chocolate before agreeing to a selfie, an insight for future studies. In this area, a human collaborator with the robot would have been ideal, to direct and manage the groups forming. In addition, our study covered a limited time-frame; longer studies may discover a different or changing effect over time, as shoppers become inured to social robots in shopping malls.

Future work shall aim to progress experiments in this area. We tested with only one type of sample - chocolates - and this may have an effect on the number and type of shoppers willing to interact. In future studies, different types of samples or products could be tested. Additionally a different type of humanoid robot could be tested or a comparison between individual operators and human / robot operators collaborating performed.

We conclude that to enable a viable commercial application, a suggested way forward should involve collaboration between a robot and human, with the robot operator in the job of attracting shoppers and the human operator working to maintain engagement and increase the number of giveaways.

\section{ACKNOWLEDGMENT}

This research is supported by an Australian Government Research Training Program Scholarship. We thank the students Jose Gunawarman and Le Kang, the University of Technology Sydney; ARC Discovery Project scheme that supports grant DP160102693; and CBA-UTS Social Robotics Partnership. 


\section{REFERENCES}

[1] B. Evangelista. Robots greet westfield mall shoppers in san francisco. [Online]. Available: http://www.sfgate.com/business/article/Robotsgreet-Westfield-mall-shoppers-in-San-10631291.php

[2] N. Woolf. Robocop is real - and could be patrolling a mall near you. [Online]. Available: https://www.theguardian.com/us-news/2016/may/20/robocoprobot-mall-security-guard-palo-alto-california

[3] S. Sabanovic, M. P. Michalowski, and R. Simmons, "Robots in the wild: Observing human-robot social interaction outside the lab," in Advanced Motion Control, 2006. 9th IEEE International Workshop on. IEEE, 2006, pp. 596-601.

[4] G. Castellano, A. Pereira, I. Leite, A. Paiva, and P. W. McOwan, "Detecting user engagement with a robot companion using task and social interaction-based features," in Proceedings of the 2009 international conference on Multimodal interfaces. ACM, 2009, pp. 119-126.

[5] Y. Murakawa, K. Okabayashi, S. Kanda, and M. Ueki, "Verification of the effectiveness of robots for sales promotion in commercial facilities," in System Integration (SII), 2011 IEEE/SICE International Symposium on. IEEE, 2011, pp. 299-305.

[6] T. Kanda, M. Shiomi, Z. Miyashita, H. Ishiguro, and N. Hagita, "An affective guide robot in a shopping mall," in Human-Robot Interaction (HRI), 2009 4th ACM/IEEE International Conference on. IEEE, 2009, pp. $173-180$.

[7] S. Satake, K. Hayashi, K. Nakatani, and T. Kanda, "Field trial of an information-providing robot in a shopping mall," in 2015 IEEE/RSJ International Conference on Intelligent Robots and Systems (IROS), Sept 2015, pp. 1832-1839.

[8] F. Dayoub, T. Morris, and P. Corke, "Rubbing shoulders with mobile service robots," IEEE Access, vol. 3, pp. 333-342, 2015.

[9] R. Ros, M. Nalin, R. Wood, P. Baxter, R. Looije, Y. Demiris, T. Belpaeme, A. Giusti, and C. Pozzi, "Child-robot interaction in the wild: Advice to the aspiring experimenter," in Proceedings of the 13th International Conference on Multimodal Interfaces, ser. ICMI ' 11. New York, NY, USA: ACM, 2011, pp. 335-342.

[10] C. Datta, A. Kapuria, and R. Vijay, "A pilot study to understand requirements of a shopping mall robot," in Human-Robot Interaction (HRI), 2011 6th ACM/IEEE International Conference on. IEEE, 2011, pp. $127-128$.

[11] S. M. Anzalone, S. Boucenna, S. Ivaldi, and M. Chetouani, "Evaluating the engagement with social robots," International Journal of Social Robotics, vol. 7, no. 4, pp. 465-478, 2015.

[12] C. Rich, B. Ponsler, A. Holroyd, and C. L. Sidner, "Recognizing engagement in human-robot interaction," in Human-Robot Interaction (HRI), 2010 5th ACM/IEEE International Conference on. IEEE, 2010, pp. 375-382.

[13] C. L. Sidner, C. Lee, C. D. Kidd, N. Lesh, and C. Rich, "Explorations in engagement for humans and robots," Artificial Intelligence, vol. 166, no. 1-2, pp. 140-164, 2005.

[14] J. Lehmann, M. Lalmas, E. Yom-Tov, and G. Dupret, "Models of user engagement," in International Conference on User Modeling, Adaptation, and Personalization. Springer, 2012, pp. 164-175.

[15] A. M. Rosenthal-von der Pütten, A. Weiss, and S. Šbanović, "The challenge (not) to go wild!: Challenges and best practices to study hri in natural interaction settings," in The Eleventh ACM/IEEE International Conference on Human Robot Interaction, ser. HRI '16. Piscataway, NJ, USA: IEEE Press, 2016, pp. 583-584.

[16] C. Shi, S. Satake, T. Kanda, and H. Ishiguro, "How would store managers employ social robots?" in 2016 11th ACM/IEEE International Conference on Human-Robot Interaction (HRI), March 2016, pp. 519520.

[17] M. E. Foster, R. Alami, O. Gestranius, O. Lemon, M. Niemelä, J.M. Odobez, and A. K. Pandey, "The mummer project: Engaging human-robot interaction in real-world public spaces," in International Conference on Social Robotics. Springer, 2016, pp. 753-763.

[18] S. Kiesler, "Fostering common ground in human-robot interaction," in Robot and Human Interactive Communication, 2005. ROMAN 2005. IEEE International Workshop on. IEEE, 2005, pp. 729-734.

[19] S. Thrun, M. Bennewitz, W. Burgard, A. B. Cremers, F. Dellaert, D. Fox, D. Hahnel, C. Rosenberg, N. Roy, J. Schulte, et al., "Minerva: A second-generation museum tour-guide robot," in Robotics and automation, 1999. Proceedings. 1999 IEEE international conference on, vol. 3. IEEE, 1999.
[20] T. Salter, K. Dautenhahn, and R. Bockhorst, "Robots moving out of the laboratory-detecting interaction levels and human contact in noisy school environments," in Robot and Human Interactive Communication, 2004. ROMAN 2004. 13th IEEE International Workshop on. IEEE, 2004, pp. 563-568.

[21] S. Duck, Human relationships. Sage, 2007.

[22] E. Hutchins, Cognition in the Wild. MIT press, 1995.

[23] L. Moshkina, S. Trickett, and J. G. Trafton, "Social engagement in public places: a tale of one robot," in Proceedings of the 2014 ACM/IEEE international conference on Human-robot interaction. ACM, 2014, pp. 382-389.

[24] C. L. Sidner, C. D. Kidd, C. Lee, and N. Lesh, "Where to look: a study of human-robot engagement," in Proceedings of the 9th international conference on Intelligent user interfaces. ACM, 2004, pp. 78-84.

[25] C. Nass and L. Gong, "Maximized modality or constrained consistency?" in AVSP'99-International Conference on Auditory-Visual Speech Processing, 1999.

[26] S. Ivaldi, S. Lefort, J. Peters, M. Chetouani, J. Provasi, and E. Zibetti, "Towards engagement models that consider individual factors in hri: On the relation of extroversion and negative attitude towards robots to gaze and speech during a human-robot assembly task," International Journal of Social Robotics, pp. 1-24, 2016.

[27] M. P. Michalowski, S. Sabanovic, and R. Simmons, "A spatial model of engagement for a social robot," in Advanced Motion Control, 2006. 9th IEEE International Workshop on. IEEE, 2006, pp. 762-767.

[28] K. Drejing, S. Thill, and P. Hemeren, "Engagement: A traceable motivational concept in human-robot interaction," in 2015 International Conference on Affective Computing and Intelligent Interaction (ACII), Sept 2015, pp. 956-961.

[29] L. J. Corrigan, C. Basedow, D. Küster, A. Kappas, C. Peters, and G. Castellano, "Perception matters! engagement in task orientated social robotics," in Robot and Human Interactive Communication (ROMAN), 2015 24th IEEE International Symposium on. IEEE, 2015, pp. 375-380.

[30] N. Memarovic, M. Langheinrich, F. Alt, I. Elhart, S. Hosio, and E. Rubegni, "Using public displays to stimulate passive engagement, active engagement, and discovery in public spaces," in Proceedings of the 4th Media Architecture Biennale Conference: Participation, ser. MAB '12. New York, NY, USA: ACM, 2012, pp. 55-64.

[31] C. Heilman, K. Lakishyk, and S. Radas, "An empirical investigation of instore sampling promotions," British Food Journal, vol. 113, no. 10, p. 12521266, Sep 2011.

[32] R. O. Gilbert, "Sen's nonparametric estimator of slope," Statistical Methods for Environmental Pollution Monitoring, John Wiley and Sons, pp. 217-219, 1987.

[33] J. Cohen, "Eta-squared and partial eta-squared in fixed factor anova designs," Educational and psychological measurement, vol. 33, no. 1, pp. 107-112, 1973.

[34] C. O. Fritz, P. E. Morris, and J. J. Richler, "Effect size estimates: current use, calculations, and interpretation." Journal of experimental psychology: General, vol. 141, no. 1, p. 2, 2012.

[35] P. Slovic and E. Peters, "Risk perception and affect," Current directions in psychological science, vol. 15, no. 6, pp. 322-325, 2006.

[36] M. Gardner and L. Steinberg, "Peer influence on risk taking, risk preference, and risky decision making in adolescence and adulthood: an experimental study." Developmental psychology, vol. 41, no. 4, p. $625,2005$.

[37] M. E. Shaw, Group dynamics: The psychology of small group behavior, 2nd ed. New York: McGraw-Hill, 1976. 